\title{
Effects of a Low Dose X-Ray Irradiation and Simulated Microgravity on Physiological, Oxidative Stress Biomarker and Metabolomics Adaptations
}

\author{
N Siddiqui ${ }^{1}$, AJ Parsian², RJ Griffin², R Pathak ${ }^{4}$, G Boysen ${ }^{3}$ and P Chowdhury ${ }^{1}$ \\ ${ }^{1}$ Department of Physiology and Cell Biology \\ ${ }^{2}$ Department of Radiation Oncology \\ ${ }^{3}$ Department of Environmental and Occupational Health, University of Arkansas for Medical Sciences, Little Rock, USA \\ ${ }^{4}$ Department of Pharmaceutical Sciences
}

\begin{abstract}
This aim of this study is to utilize a ground-based rat model of hindlimb unloading and examine the effect of 0.3 GY radiation on oxidative stress biomarker and metabolomics adaptations. Methods: For this study, we have used four groups (four rats per group) for three weeks in the following order: 1) non-suspended control (CON), 2) irradiated non-suspended (IR), 3) irradiated plus hindlimb suspended (IR+HLS) and 4) hindlimb suspended (HLS). IR and IR+HLS groups were exposed to five sessions of X-ray irradiation (0.3 Gy each daily). Body weight, food and water intakes measured daily. At term, the animals were anesthetized with isoflurane, blood was drawn in a syringe to collect serum for protein and metabolomics assays. Lipid peroxidation was measured on collected tissue samples. Data were pooled, analyzed and tested statistically using one way ANOVA. Results: CON animals gained weight faster than IR + HLS or HLS group, whereas weight gain in IR group was closely similar to control group. Food consumption and water intakes were not significantly different between the groups. Serum insulin levels and tissue MDA levels were variable among the groups with no significant differences. Serum metabolites showed significant differences in aspartate and gammaglutamyl-glutamine concentrations between HLS and IR + HLS groups as compared to control groups although many of the other metabolomes measured were not significantly changed. Summary: HLS and HLS +IR animals showed reduced growth as compared to control and IR groups. There are insignificant but variable changes in tissue oxidative parameters and insulin levels. Significant changes in some specific serum metabolites were observed. Conclusions: Decreased body weight gain in HLS and HLS + IR groups do not appear to be due to decrease in food intake. Changes in specific metabolites in HLS and HLS + IR group appear to be a stress related response. Overall, the data reflects variability due to altered experimental design and low radiation effect (Supported by a grant from Arkansas Space Grant Consortium).
\end{abstract}

KEYWORDS: Hindlimb suspension; Oxidative stress; X-irradiation; Metabolomics changes; Tissue malondialdehyde levels

ABBREVATIONS: IR: Irradiated; HLS: Hindlimb Suspended; GT: Glutathione; MDA: Malondialdehyde; SOD: Superoxide Dismutase; HLU: Hindlimb Unloaded

Quick Response Code:

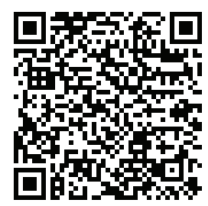

Address for correspondence: Parimal Chowdhury, Department of Cellular Physiology and Molecular Biophysics, Professor Emeritus, College of Medicine, University of Arkansas for Medical Sciences, USA

Received: September 21, $2021 \quad$ Published: October 08, 2021

How to cite this article: Siddiqui N, Parsian AJ, Griffin RJ, Rupak P, Boysen G, Chowdhury P. Plummer Vinson Syndrome: First Two Tunisian Pediatric Cases. 2021- 3(5) OAJBS.ID.000330. DOI: $10.38125 / \mathrm{OAJBS} .000330$ 


\section{INTRODUCTION}

Imbalances in oxidant-antioxidant defense systems of the body can occur due to severe oxidative stress development, which has been documented during space flight and in aging $[1,2]$. Oxidative stress during aging is attributed to a generalized increase in free radical production, causing a decrease in antioxidant activity. These physiologic alterations are quantifiable by assays for malondialdehyde (MDA), superoxide dismutase (SOD) and glutathione (GT) assays. Observation of human subjects [3] and studies in animals [4-8] exposed to sub-lethal doses of irradiation (IR) had identified anorexia as one of common components of acute radiation injury syndrome suggesting that it could also contribute to development of space flight anorexia and weight loss [3]. The objective of the current study is to examine the induced oxidative parameters in this rodent model of simulated microgravity and to determine the effects of a low dose X-ray Irradiation and simulated microgravity on physiological, oxidative stress biomarker and metabolomics adaptations.

MDA is a reactive aldehyde and known biomarker for oxidative stress [9-11], produced by the free radical-induced decomposition of polyunsaturated fatty acids into lipid peroxides. Oxidative stress induces an increase in oxidation of membrane fatty acid moieties leading to production of unstable lipid peroxides that degrade rapidly into products such as malondialdehyde [12]. The formation of these aldehydes in cells contributes to the mechanisms of oxidantinduced injury, including permeabilization of cell membranes and membranes of organelles such as lysosomes, which can activate apoptotic pathways [13]. Reduced glutathione content in the tissues is also an indicator for oxidative stress [14] for it plays a key role in the detoxification of cells by reacting with hydrogen peroxide and organic peroxides. In this study, we evaluated the influence of hind limb suspension on tissue oxidative stress in selected organs isolated from rats after sacrifice. Earlier studies from our laboratory used a NASA validated rat hindlimb-unloaded (HLU) model of exposure to microgravity $[15,16]$. In this study, we have studied the effect of low dose radiation on various parameters utilizing a modified hindlimb unloading (HLU) system. The low dose of radiation used in the current study is from an earlier study published from our laboratory recently [17].

\section{MATERIALS AND METHODS}

Adult male Sprague-Dawley rats (Charles River Laboratories, Inc.) were use in this study. All animals had free access to food pellets and water. Body weight was measured daily until sacrifice. Food and water intake were also measured daily until sacrifice. Following one-week acclimation period in Department of Laboratory Animal Medicine care facilities, the animals were divided into four groupsControl (CON), irradiated (IR), irradiated and hindlimb suspended (IR + HLS), and hindlimb suspended (HLS). Sixteen animals, four animals in each group $(n=4)$ were used in the study.

\section{Hindlimb Suspension}

On day of initial suspension, rats in HLS and HLS + IR groups were anesthetized via isoflurane inhalation for 3-4 minutes and fitted with tail harnesses. The harness consisted of a strip of SkinTrac adhesive foam (Zimmer, Inc., Charlotte, NC), cut in half and applied on the dorsal and ventral surfaces of the tail, a wrapping of medical gauze around the Skin-Trac, and pieces of glass tape to secure both layers together. A ball bearing chain was taped to the dorsal surface of the harness and fitted through a key ring, which was placed on a metal bar running along the top of the custom suspension enclosure, allowing the animals to be suspended at a 30-degree angle with the head tilted downward. This ensured that their hind legs would not be able to make contact with the floor of the cage and induced a net shift in total body fluid towards the cephalic region. After three weeks all animals sacrificed to collect blood and tissues samples.

\section{Radiation}

Rats from the IR and HLS + IR groups were subjected to 0.3 Gy of X-Ray irradiation 5 days per week, for all three weeks of the experimental duration. The animals were transported to the irradiation facility between 1:00 PM and 2:00 PM each day. A Faxitron cabinet X-ray system (Lincolnshire, IL, USA) was used to provide a fixed dose of irradiation exposure.

\section{Sacrifice and Tissue Collection}

Upon completion of the 3-week experimental period, all animals were euthanized over a period of 2 days. Prior to sacrifice, animals were deeply anesthetized via isoflurane inhalation, followed by cardiac puncture and exsanguination to collect blood samples. From each animal, brain, lungs, heart, quadriceps muscle, duodenum of the small intestine, descending colon, liver, kidneys, testes, and pancreas were collected. Harvested tissues were washed in $0.9 \%$ $\mathrm{NaCl}$ solution, and $\leqq 1$ gram was utilized from each tissue, were homogenized in ice-cold $20 \mathrm{mM}$ Tris- $\mathrm{HCl}$ buffer, $\mathrm{pH}=7.4$ (tissue to buffer ratio, $1: 10 \mathrm{w} / \mathrm{v}$ ). $10 \mu \mathrm{L}$ of $0.1 \%$ butylated hydroxytoluene (BHT) was added to each homogenate to prevent further formation of oxidative species. In addition, 3.8\% citrate was added to blood samples collected from each animal (citrate to blood ratio, 1:10 $\mathrm{v} / \mathrm{v}$ ), which was then spun down at 8,000 rpm to collect serum.

\section{MDA}

All tissue homogenates and serum samples were analyzed for levels of malondialdehyde (MDA) production as a biomarker of oxidative stress, using the LPO-586 assay (Bioxy tech LPO-586, R\&D Systems, Minneapolis, MN 55413). MDA can be detected in vitro by reacting it with a chromogenic reagent, 1-methyl-2phenylindole, at 45 ? for one hour to yield a stable chromophore, levels of which can then be quantified via UV-Vis spectroscopy at an absorbance of $586 \mathrm{~nm}$. Results were plotted against a standard curve which was developed by using varying concentrations of 1, 1, 3, 3-tetramethoxypropane. MDA levels, expressed as $\mu \mathrm{M}$ concentrations, were normalized to protein concentrations for each sample $(\mu \mathrm{M} / \mu \mathrm{g})$.

\section{Protein}

Total protein levels for all collected tissue homogenates and serum samples were quantitated in $\mu \mathrm{g} / \mu \mathrm{L}$ via Bradford assay (BioRad Laboratories), using bovine serum albumin concentrations as standards.

\section{Insulin ELISA}

Upon termination of the study, serum samples were collected from each animal. The samples were analyzed for differential insulin levels, as expressed in nanograms per milliliter, using an ultra-sensitive ELISA assay (\#90060, Crystal Chem Inc., USA).

\section{Metabolomics}

Serum samples from each animal were analyzed for the presence of over 100 metabolites using mass spectrometry. 


\section{Statistical Analysis}

All data were analyzed using Prism software (version 8.0 Graph Pad). Results are expressed as mean \pm standard deviation (SD). Experimental endpoint measurements were compared via 1-way analysis of variance (ANOVA). Continuous measurements were compared via 2-way ANOVA.

\section{RESULTS}

\section{Physical Data [Figures 1-6]}

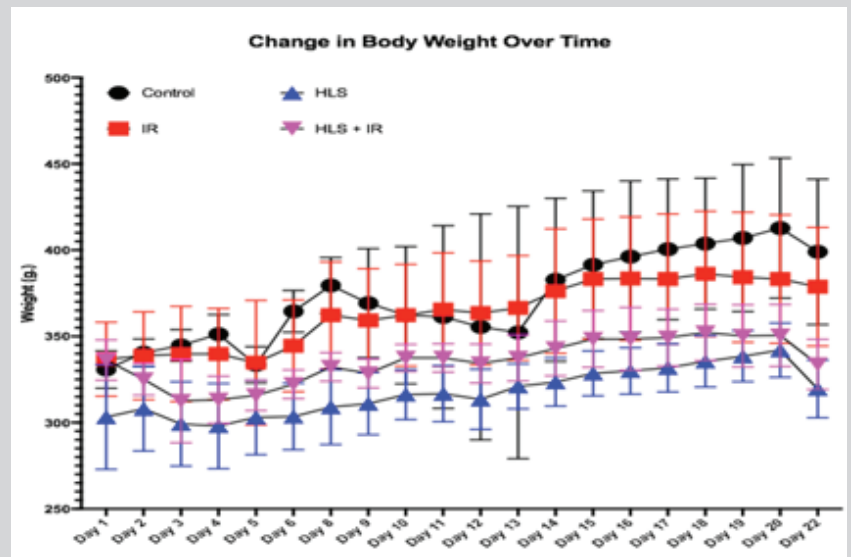

Figure 1: Body weight changes during suspension period; IR- irradiated; HLS- hindlimb suspended; HLS + IR- hindlimb suspended and irradiated.

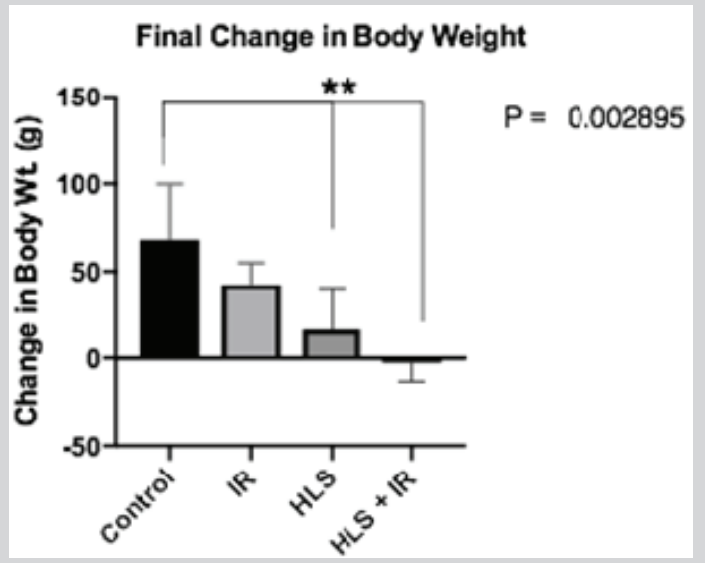

Figure 2: Final body weight change during suspension period; IR- irradiated; HLS- hindlimb sUspended; HLS + IRhindlimb suspended and irradiated.

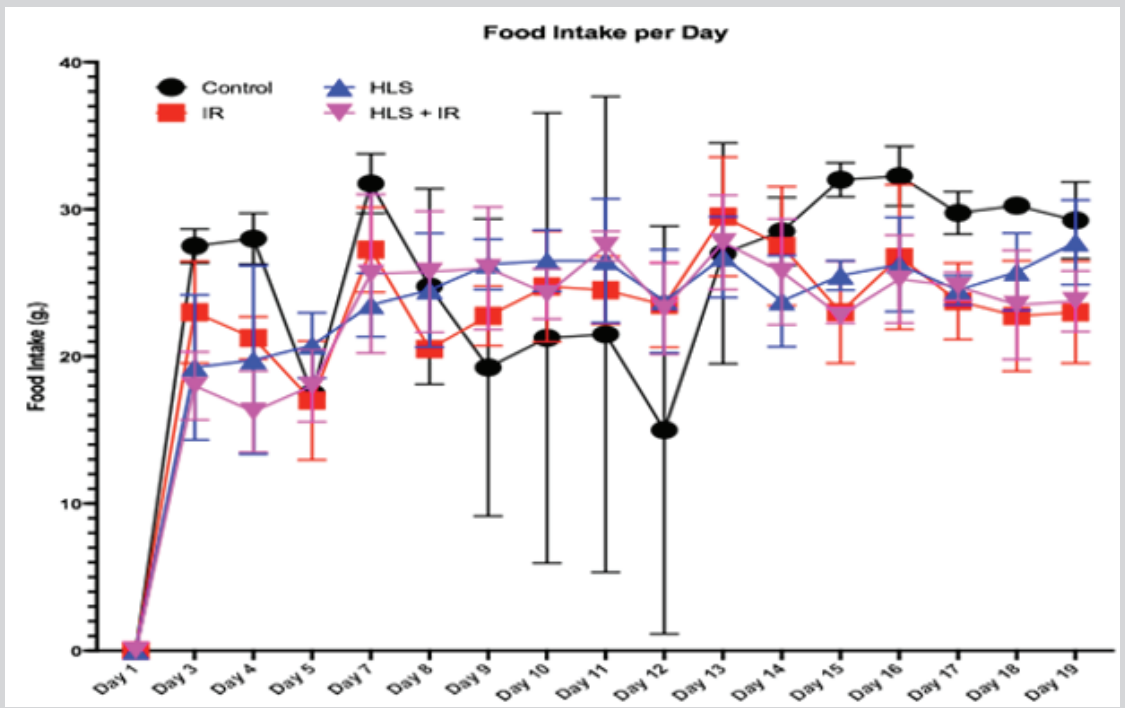

Figure 3: Average food consumption per day for all groups during suspension period; IR- irradiated; HLS- hindlimb suspended; HLS + IR- hindlimb suspended and irradiated. 


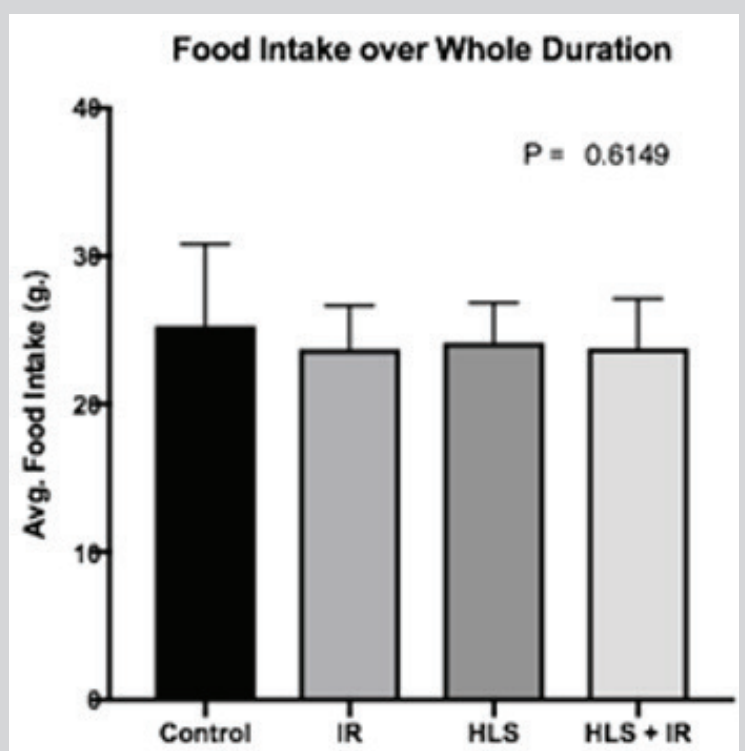

Figure 4: Final change in food intake for all groups during suspension period; IR- irradiated; HLS- hindlimb suspended; HLS + IR- hindlimb suspended and irradiated.

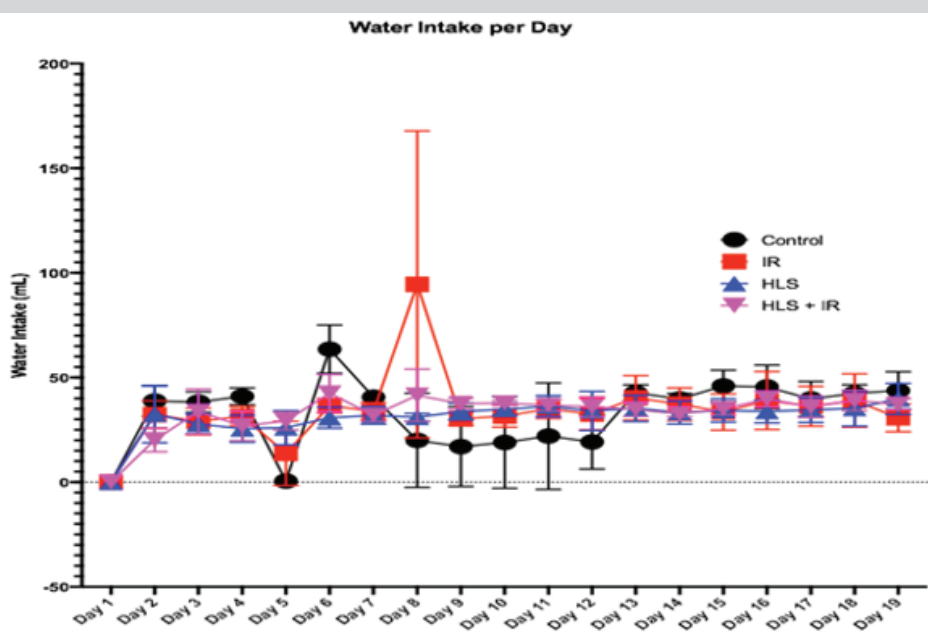

Figure 5: Average water intake per day for all groups during suspension period; IR- irradiated; HLS- hindlimb suspended; HLS + IR- hindlimb suspended and irradiated.

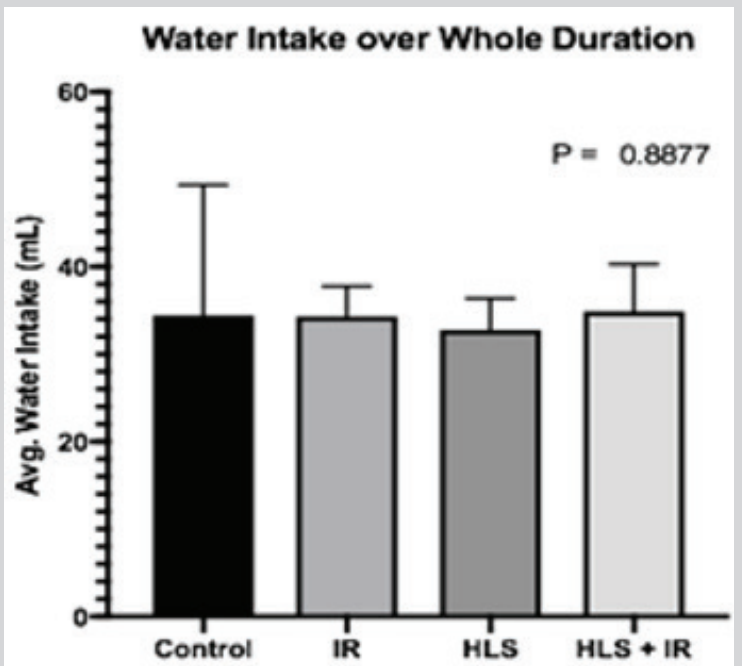

Figure 6: Final change in water intake for all groups during suspension period; IR- irradiated; HLS- hindlimb suspended; HLS + IR- hindlimb suspended and irradiated. 


\section{Physiological Assay Data [Figures 7-8]}
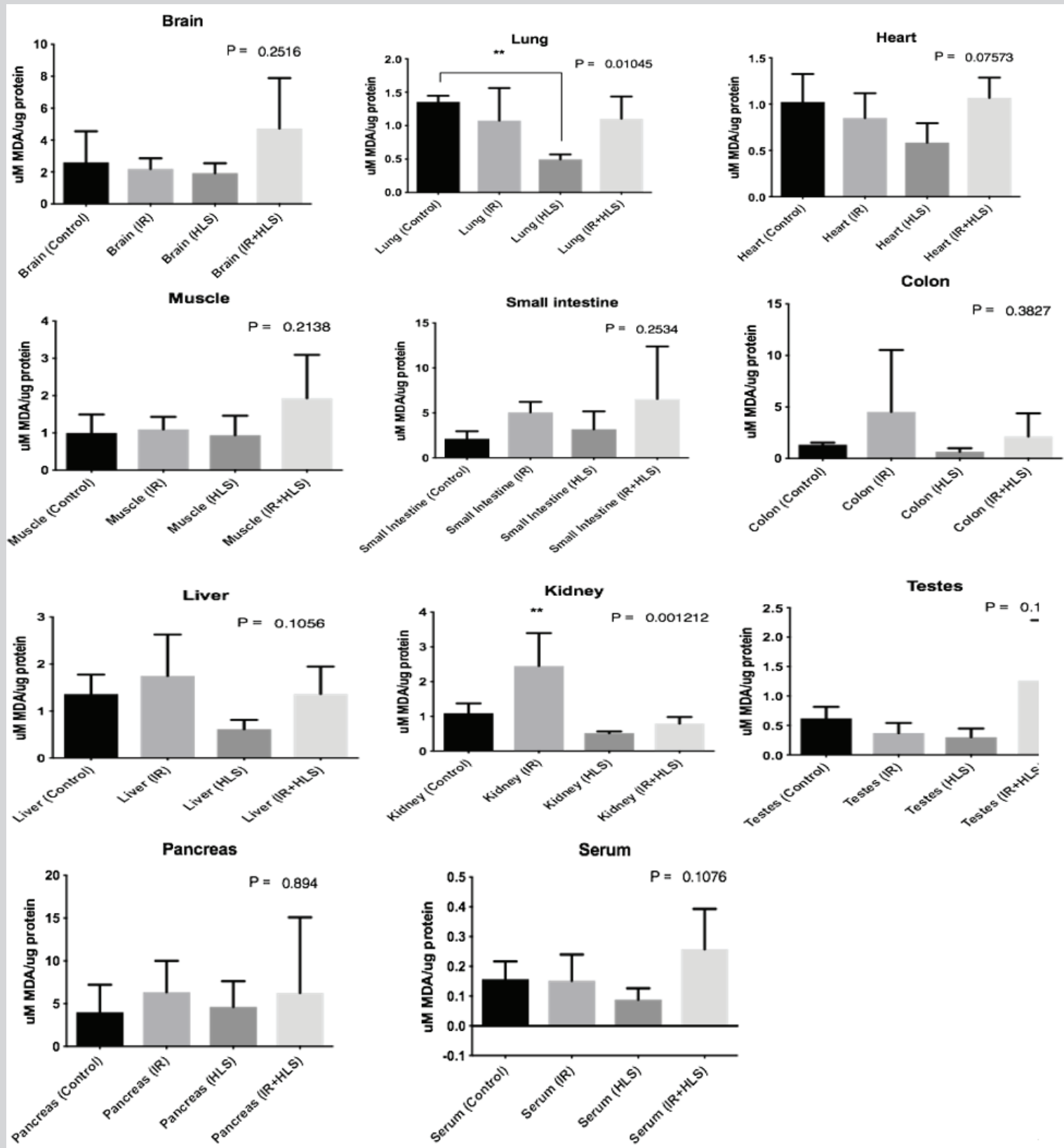

Figure 7: MDA production levels normalized to protein concentration for tissues collected $(\mu \mathrm{M} / \mu \mathrm{g})$ in all groups, plus serum; IR- irradiated; HLS- hindlimb suspended; HLS + IR- hindlimb suspended and irradiated.

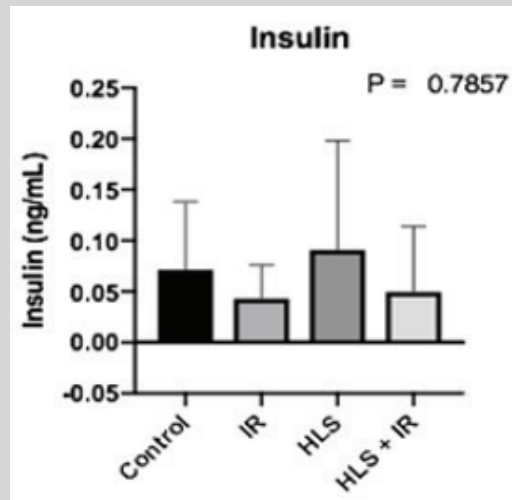

Figure 8: Serum insulin levels among all 4 groups; IR- irradiated; HLS- hindlimb suspended; HLS + IR- hindlimb suspended and irradiated. 


\section{Metabolomics Data [Figure 9]}

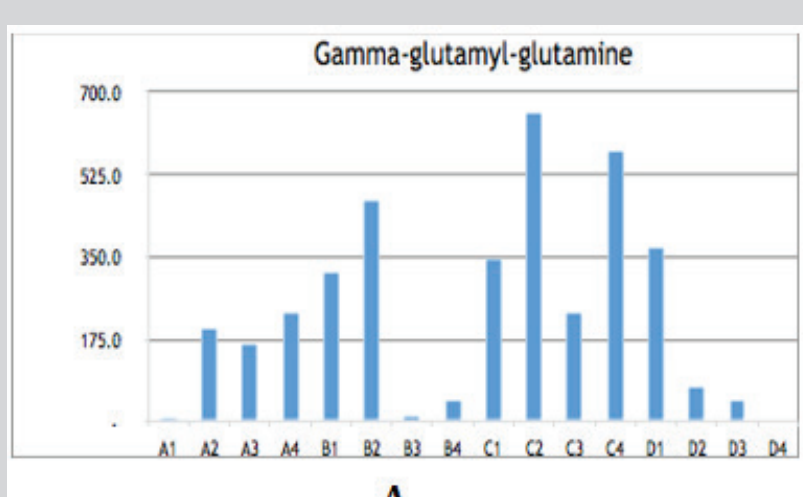

A

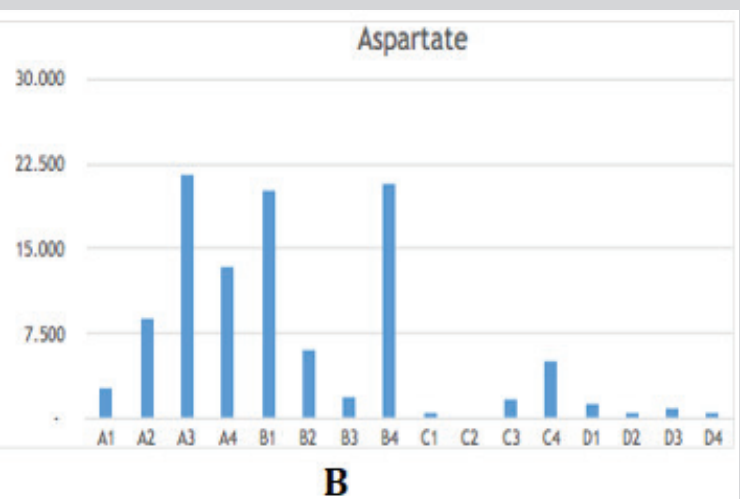

B

Figure 9: A) Individual serum levels of gamma-glutamyl-glutamine among all 16 animals; B) Individual serum levels of aspartate among all 16 animals; CON (A1, A2, A3, A4), IR (B1, B2, B3, B4), HLS (C1, C2, C3, C4), HLS + IR (D1, D2, D3, D4).

\section{SUMMARY OF RESULTS}

As shown in Figures 1 and 2, the rate of body weight increases of animals in the suspended and irradiated plus suspended groups decreased significantly as compared to control or irradiated group. There was no change in food intake (Figures 3 and 4) or water intake (Figures 5 and 6) among the groups. The results of tissue MDA levels are shown in Figure 7. There is a wide variation of MDA levels in various tissues, and it is consistent in all groups. The variation in tissue MDA levels noted in this study may be due to experimental complexity as encountered during the experiment, such as temporary de-suspension during radiation treatment and loss of suspension during the maintenance period. Serum insulin levels also varied among different groups, but it is elevated when suspension and radiation were combined as shown in Figure 8. Serum metabolome profile as measured by mass spectrometry showed significant differences in aspartate and gamma-glutamylglutamine concentrations between HLS and IR + HLS groups as compared to control groups although many of the other metabolomes measured were not significantly changed (Figure 9).

\section{DISCUSSION}

It is unclear whether exposure to low dose radiation and microgravity during space flight act either independently or in concert to produce complications such as anorexia, body weight, and muscle and bone loss during a space flight. As a first step of addressing this question, we evaluated the effects of relatively high doses of X-ray irradiation (five exposures of 1.2 Gy each) on food and water consumption, body weight, BMD, and soleus muscle weight of normal control and HLS rats (ground-based model of microgravity) [17]. These types of radiation doses are likely to yield on the high end of what would occur during a particularly active space flight with regard to solar particle events. However, our studies suggest that, at least with regard to the effect on food consumption, there is no safe dose of the exposure and that noticeable effects on body weight could also be observed at space flight relevant exposures (0.3-0.5 Gy). In light of this finding, studies using other space flight relevant radiation exposure regimens such as mixed particle, longer exposure times or lower exposure rates are warranted to further delineate the potential association of space irradiation and microgravity on weight gain, food intake, and musculoskeletal atrophy.
In analyzing the effects of experimental microgravity, we confirmed previous observations of deteriorating effects of HLS on rat body weight [15-18]. We have also observed only a small but transient net weight loss of HLS animals with no effects of HLS on mean daily water or food intake. These observations are also consistent with previous data, showing that when detected, such changes are small in magnitude and constrained to a few days that are required for the animal to adapt to a new environment of HLS [19-21]. In general, our study is also in agreement with previous studies of the effects of irradiation on weight and food consumption by rats, confirming that a relatively low dose of whole-body IR (0.3GY) results in acute reduction in both food intake and weight loss [17]. The major difference in those reports and our study is the timing of onset and duration of post-irradiation changes in the studied parameters.

Despite the experimental variability there is insignificant change in tissue oxidation. This was understandable based on the fact that multiple low-dose irradiation of mice reduced inflammatory signaling in a mouse model of asthma [22] which suggest that low doses of radiation have an effect that is opposite of that from high doses in atherosclerosis development, and that dose rate is a very important parameter to consider. Furthermore, dose rate dependence of inflammatory response and endothelial cell function has been demonstrated recently in published in vitro experiments [23]. These studies provide strong emphasis in induced increase in anti-inflammatory and anti-oxidative parameters due to chronic radiation as shown in atherosclerotic plaque size reduction in ApoE -/- mice [24].

The enzyme GGTP participates in the transfer of amino acids and peptides across the cellular membrane and possibly participates in glutathione metabolism. The highest concentrations of this enzyme are found in the liver and biliary tract. Smaller amounts are found in the kidney, spleen, heart, intestine, brain, and prostate gland. GGT is concentrated in the liver, but it is also present in the gallbladder, spleen, pancreas, and kidneys. GGT blood levels are usually high when the liver is damaged [25]. We have found high levels of aspartate and GGT in the serum of these animals with HLS suggesting an altered function of the liver. These effects are reduced when animals are exposed to radiation demonstrating the anti -inflammatory and anti-oxidative effects. More data will need to be collected to ensure these effects. 


\section{ACKNOWLEDGEMENTS}

This study was supported by a grant from the Arkansas Space Grant Consortium. (NNX15AR71H)

\section{REFERENCES}

1. Stein T (2002) Space flight and oxidative stress. Nutrition 18: 867-871.

2. Afanas'ev I (2009) Superoxide and nitric oxide in senescence and aging. Front Biosci 14: 3899-3912.

3. Da Silva MS, Zimmerman PM, Meguid MM, Nandi J, Ohinata K, et al. (2002) Anorexia in space and possible etiologies: an overview. Nutrition 18: $805-813$

4. Garcia J, Kimeldorf DG, Hunt EL, Davies BP (1956) Food and water consumption of rats during exposure to gamma-radiantion. Radiat Res 4: 33-41.

5. Kandasamy SB (1998) Effect of ionizing radiation on the release of cholecystokinin in the hypothalamus of the rat. Radiat Res 150: 298-303.

6. Smith DE, Tyree EB (1956) Influence of x-irradiation upon water consumption by the rat. Am J Physiol 184: 127-133.

7. Varma M, Sato T, Zhang L, Meguid MM (2000) Space flight re 7 lated anorexia. Lancet 356: 681 .

8. Winsauer PJ, Verrees JF, Halloran KP, Bixler MA, Mele PC, et al. (1994) Effects of chlordiazepoxide, 8-OH-DPAT and ondanse 7 tron on radiationinduced decreases in food intake in rats. J Pharmacol Exp Ther 270(1): 142-149.

9. Matsushima Y, Nanri H, Nara S, Okufuji T, Ohta M, et al. (2006) Hindlimb unloading decreases thioredoxin-related antioxidant proteins and increases thioredoxin-binding protein-2 in rat skeletal muscle. Free Radic Res 40: 715-722.

10. Sui P, Pistilli E, Always S (2008) Age-dependent increase in oxidative stress in gastrocnemius muscle with unloading. J Appl Physiol 105 1695-1705.

11. Lui M, Li X, Guo H, Lee K, Qin L, et al. (2003) The Effects of verbascoside on plasma lipid peroxidation level and erythrocyte membrane fluidity during immobilization in Rabbits. Life Sciences 73: 883-892.

12. Esterbauer H, Schaur R, Zollner H (1991) Chemistry and biochemistry of 4-hyroxynonenal, malondaldehyde and related aldehydes. Free Radic Biol Med 11: 81-128.

13. Aits S, Jäättelä M (2013) Lysosomal cell death at a glance. J Cell Sci 126(1): 1905-1912.

14. Fang Y, Sun C, Tian X, Cong J (1998) Effect of Lu-duo-wei on scavenging superoxide and hydroxyl radicals in vitro. Am J Chin Med 26: 153-158.
15. Chowdhury P, Soulsby M, Kim K (2007) L-carnitine influence on oxidative stress induced by hind-limb unloading in adult rats. Aviat Space Environ Med 78: 554-556.

16. Chowdhury P, Soulsby M, Scott J (2009) Effects of aminoguanidine on tissue oxidative stress induced by hind-limb unloading in rats. Ann Clin Lab Sci 39(1): 64-70.

17. Chowdhury P, Akel N, Jamshidi PA, Gaddy D, Griffin RJ, et al. (2016) Degenerative tissue responses to space like radiation doses in rodent model of simulated microgravity. Ann Clin \& Lab Sci 46(2): 190-197.

18. Chowdhury P, Soulsby M (2008) Oxidant/Antioxidant status in rats exposed to simulated weightlessness by Hind-limb unloading and reloading. The Open Clinical Chemistry J 1: 47-56.

19. Chowdhury P, Long A, Harris G, Soulsby ME, Dobretsov M, et al. (2013) Animal model of simulated microgravity: a comparative study of hindlimb unloading via tail versus pelvic suspension. Physiological Reports 1: e00012.

20. Da Silva MS, Zimmerman PM, Meguid MM, Nandi J, Ohinata K, et al. (2002) Anorexia in space and possible etiologies: an overview. Nutrition 18(10): 805-813.

21. Morey Holton E, Globus RK, Kaplansky A, Durnova G (2005) The hindlimb unloading rat model: literature overview, tech $\neg$ nique update and comparison with space flight data. Adv Space Biol Med 10: 7-40.

22. Lodermann B, Wunderlich R, Frey S, Schorn C, Stangl S, et al. (2012) Low dose ionizing radiation leads to a NF-kappaB dependent decreased secretion of active IL-1beta by activated macrophages with a discontinuous dose-dependency. Int J Radiat Biol 88: 727-734.

23. Ebrahimian T, Le Gallic C, Stefani J, Dublineau I, Yentrapalli R, et al. (2015) Chronic gamma-irradiation induces a dose-rate-dependent pro-inflammatory response and associated loss of function in human umbilical vein endothelial cells. Radiat Res 183: 447-454.

24. Ebrahiminian TG, Beugnies L, Surette J, Priest N, Gueguen Y, et al. (2018) Chronic exposure to external low dose gamma radiation induces an increase in anti-inflammatory and anti-oxidative parameters resulting in atherosclerotic plaque size reduction in ApoE -/- mice. Radiat Res 189(2): 187-196.

25. Rekha M, Jayaprakash MDS (2011) Assessment of serum enzymes gamma glutamyl transferase, aspartate transaminase and alanine transaminase in liver diseases. J Pharm Sci \& Res 3(5): 1221-1226.

26. Sappington DR, Siegel ER, Hiatt G, Desai A, Penney RB, et al. (2016) Glutamine drives glutathione synthesis and contributes to radiation sensitivity of A549 and H460 lung cancer cell lines. Biochim Biophys Acta 1860(4): 836-843. 